\title{
Uso de medicamentos e fatores associados à polifarmácia em indivíduos com diabetes mellitus em Minas Gerais, Brasil
}

\author{
Drug utilization and factors associated with polypharmacy \\ in individuals with diabetes mellitus in Minas Gerais, Brazil
}

\author{
Michael Ruberson Ribeiro da Silva ${ }^{1}$ \\ Leonardo Maurício Diniz ${ }^{2}$ \\ Jéssica Barreto Ribeiro dos Santos ${ }^{1}$ \\ Edna Afonso Reis ${ }^{3}$ \\ Adriana Rodrigues da Mata ${ }^{1}$ \\ Vânia Eloisa de Araújo ${ }^{4}$ \\ Juliana Álvares ${ }^{1}$ \\ Francisco de Assis Acurcio ${ }^{1}$
}

${ }^{1}$ Departamento de Farmácia Social, Faculdade de Farmácia, Universidade Federal de Minas Gerais (UFMG). Av. Antônio

Carlos 6627, Pampulha. 31270-901 Belo Horizonte MG Brasil.

mruberson@gmail.com

${ }^{2}$ Departamento de Clínica

Médica, UFMG. Belo

Horizonte MG Brasil.

${ }^{3}$ Departamento de

Estatística, UFMG. Belo

Horizonte MG Brasil.

${ }^{4}$ Departamento de

Odontologia, Pontifícia

Universidade Católica

de Minas Gerais. Belo

Horizonte MG Brasil.
Abstract The objective of this study was to evaluate the use of drugs and the factors associated with polypharmacy in patients with diabetes mellitus (DM) in Minas Gerais. Descriptive analysis of drugs in use and logistic regression to estimate the association between socio-demographic and clinical characteristics with polypharmacy were performed. Of the 2619 respondents, 56.5\% were in polypharmacy. Drugs for DM, agent in renin-angiotensin system, and diuretics are the most frequently used. Factors such as age, comorbidities and increased access to health services were associated with polypharmacy. It was observed high prevalence of polypharmacy, which requires a suitable care and better quality of drug use in this population.

Key words Diabetes mellitus, Drug utilization, Polypharmacy, Pharmacoepidemiology
Resumo O objetivo deste estudo foi avaliar o uso de medicamentos, a prevalência e os fatores associados à polifarmácia em pacientes com diabetes mellitus (DM) em Minas Gerais. Realizou-se um estudo transversal com descrição dos medicamentos em uso e análise da associação entre características sociodemográficas e clinicas com polifarmácia, por meio de regressão logística. Dos 2619 entrevistados, 56,5\% estavam em polifarmácia. Medicamentos para DM, agentes no sistema renina-angiotensina e diuréticos foram os mais usados. Fatores como envelhecimento, presença de comorbidades e maior acesso aos serviços de saúde foram associados à polifarmácia. Observou-se elevada prevalência de polifarmácia, o que requer um cuidado adequado e melhor qualidade do uso de medicamentos para essa população.

Palavras-chave Diabetes mellitus, Uso de medicamentos, Polimedicação, Farmacoepidemiologia 


\section{Introdução}

Diabetes Melitus é uma doença crônica complexa que exige assistência médica contínua e o uso de estratégias de redução de risco multifatoriais além da busca pelo controle glicêmico ${ }^{1}$. Apresenta-se como uma epidemia mundial, sendo um grande desafio para os sistemas de saúde de todo o mundo ${ }^{2,3}$.

A prevalência mundial do DM é estimada em 387 milhões de pessoas, sendo esperado um número de 592 milhões para 2035, entre 20 e 79 anos $^{4}$. No Brasil, a prevalência do DM atinge $8,7 \%$ da população acima dos 20 anos, o que corresponde a 11,6 milhões de pessoas. Destes, 3,2 milhões seriam não diagnosticados ${ }^{5}$. Cerca de 75\% dos casos de DM tipo 1 são diagnosticados em indivíduos com idade inferior a 18 anos. Além disso, estima-se uma incidência de DM tipo 2 de 2,3\% ao ano em indivíduos com menos de 20 anos, o que aumentará significativamente a prevalência da doença nesta faixa etária da população ${ }^{6}$. Por outro lado, configura-se como uma condição de saúde importante no processo de envelhecimento, pois $26 \%$ dos pacientes com idade acima de 65 anos têm DM, e esse número deve crescer rapidamente nas próximas décadas. Os indivíduos mais velhos com diabetes têm maiores taxas de morte prematura, incapacidade funcional e doenças coexistentes do que indivíduos sem $\mathrm{DM}^{7}$, o que leva a diminuição da sua qualidade de vida ${ }^{8}$.

Nesse contexto, pacientes com DM têm maior risco de apresentarem polifarmácia do que outros pacientes ${ }^{7,9}$, definida pela maioria dos estudos como o uso de cinco ou mais medicamentos ${ }^{10-12}$. A prevalência de polifarmácia em indivíduos idosos nos Estados Unidos aumentou significativamente de $30,6 \%$ para $35,8 \%$ e o risco de interação medicamentosa maior cresceu de $8,4 \%$ para $15,1 \%$, em um período de cinco anos. Estes resultados tornam-se ainda mais relevantes ao se observar o aumento do consumo de suplementos alimentares nessa população, como vitaminas e ômega-3, o que sugere a necessidade de um maior cuidado e acompanhamento dos pacientes em uso de múltiplos medicamentos e com doenças crônicas ${ }^{10}$. Observa-se, portanto, uma crescente preocupação com a polifarmácia e o seu impacto sobre a morbidade, a mortalidade e os custos relacionados ao paciente e aos Serviços de Saúde ${ }^{13}$.

Estudos recentes em diferentes populações de pacientes com DM avaliaram a prevalência da doença ${ }^{14-18}$ e demonstraram o seu aumento nas últimas décadas, com estimativas indicando que este aumento permanecerá ao longo dos anos ${ }^{16-}$ ${ }^{18}$. Outros estudos relatam que o número de medicamentos em uso é elevado ${ }^{14,19,20}$. Entretanto, não foram observados estudos que avaliassem os fatores associados à polifarmácia na população com DM. Logo, este estudo tem por objetivo avaliar a prevalência e os fatores associados à polifarmácia e descrever o perfil de utilização de medicamentos em pacientes com diabetes melitus em Minas Gerais, Brasil.

\section{Métodos}

\section{Amostra}

Este estudo integra o projeto Dia a Dia, uma avaliação epidemiológica e econômica dos esquemas terapêuticos utilizados no tratamento do diabetes melitus em Minas Gerais. Realizou-se um inquérito domiciliar, em 63 municípios de Minas Gerais, sendo 61 do interior, Belo Horizonte e Contagem, que possuíam serviços de Assistência Farmacêutica estruturados e em funcionamento, entre janeiro e fevereiro de 2014. Os critérios para a seleção por conveniência dos municípios do interior foram: população urbana acima de 2 mil habitantes, ter unidade da Rede Farmácia de Minas em atividade há pelo menos 2 anos, possuir acesso à internet no serviço, apresentar anuência do Secretário Municipal de Saúde e manifestação de interesse do farmacêutico local em participar do projeto. A população do estudo foi constituída por indivíduos portadores de diabetes melitus, sem restrição de idade ou sexo, que concordaram em participar e assinaram o Termo de Consentimento Livre e Esclarecido (TCLE). Os municípios selecionados estão inseridos em todas as 13 macrorregiões de saúde do Estado de Minas Gerais.

A amostra para cada município foi selecionada de forma aleatória, considerando-se os setores censitários do Instituto Brasileiro de Geografia e Estatística (IBGE) no perímetro urbano. Os pesquisadores receberam mapas de localização referentes aos setores selecionados, que foram percorridos casa a casa, sem retorno na situação de morador ausente conforme os agrupamentos a seguir: a) para Belo Horizonte e Contagem, com populações de 2,4 milhões e 650 mil habitantes ${ }^{21}$, respectivamente, foram sorteados 122 e 25 setores censitários; b) para municípios com população urbana superior a 5.000 habitantes e um mínimo de dez setores censitários do IBGE, foram 
sorteados cinco setores; c) para municípios com população urbana entre 2.000 e 5.000 habitantes e com menos de dez setores censitários do IBGE, todas as casas possíveis foram visitadas.

O cálculo do tamanho amostral foi realizado considerando a prevalência de $8,7 \%$ de DM sobre a população de referência de Minas Gerais em 2013, de 20.593.366 21 , que correspondeu à estimativa de 1.791.623 indivíduos com DM, nível de confiança de $95 \%$, prevalência estimada de $50 \%$ para diferentes desfechos de prevalência do estudo, erro máximo tolerado de $2 \%$. A partir desses parâmetros, a amostra mínima final foi estimada em 2.398 indivíduos, à qual se acrescentou $10 \%$ para cobrir possíveis perdas, totalizando 2.638 indivíduos a serem entrevistados.

\section{Variáveis do estudo}

A variável dependente foi a ocorrência de polifarmácia, definida como o uso de cinco ou mais medicamentos, obtido a partir da pergunta: "Nos últimos 15 dias você utilizou medicamentos?" e comprovado com a apresentação da embalagem ou prescrição.

As variáveis independentes foram sexo, faixa etária, estado civil, escolaridade, raça, autopercepção de saúde, tipo de DM (1 ou 2) autorreferido, tempo médio de diagnóstico do DM, número de comorbidades autorreferidas, frequência de consultas médicas no último ano, tipo de assistência (pública ou plano de saúde privado), prática regular de atividade física e interrupção das atividades habituais nos últimos 15 dias.

\section{Análise dos dados}

A análise descritiva foi realizada por distribuição de frequências dos medicamentos em uso, conforme classificação anatômica terapêutica (ATC) em primeiro, segundo e quinto nível para toda a amostra. Para a variável número de medicamentos foi obtida a média e o desvio padrão.

Os fatores associados à polifarmácia foram analisados, constituindo-se dois grupos de comparação: sem polifarmácia (grupo de referência), definido como o uso de zero a quatro medicamentos, e polifarmácia, definido como o uso de cinco ou mais medicamentos. Foi realizada análise bivariada para comparação de frequências, utilizando-se o teste do qui-quadrado de Pearson. As variáveis que apresentaram valor de $\mathrm{p}<0,20$ para associação com a variável dependente na análise bivariada foram incluídas na análise multivariável, utilizando-se o modelo de regressão logística múltipla com seleção de variáveis pelo método backward. Permaneceram no modelo final apenas as variáveis associadas com valor de $\mathrm{p}<0,05$. A análise dos dados foi realizada utilizando-se o programa SPSS ${ }^{\oplus}$ versão 22.0.

\section{Considerações éticas}

O estudo foi aprovado pelo Comitê de Ética em Pesquisa da Universidade Federal de Minas Gerais.

\section{Resultados}

Foram entrevistadas 2.619 pessoas com DM, sendo que $2.192(83,7 \%)$ declararam ter DM tipo 2 e $272(10,4 \%)$ ter DM tipo 1. Cerca de 6\% não souberam relatar o tipo de DM apresentado. A idade média foi de 61,3 anos - desvio-padrão de 16,4 anos. O tempo médio de diagnóstico do DM foi de $9,9 \pm 8,8$ anos.

Considerando-se todos os participantes, foi relatado o consumo de 13.629 medicamentos para o tratamento de diversas doenças, com amplitude de zero a 17 medicamentos em uso por pessoa, conforme Gráfico 1 . O número médio de medicamentos utilizados foi de 5,2 $\pm 2,9$.

Cerca de $60 \%$ dos medicamentos foram adquiridos em farmácias públicas e 35\% eram genéricos. Os fármacos com ação no sistema cardiovascular foram os mais utilizados, dentro dos 13.629, seguido dos fármacos com ação no trato alimentar e metabolismo, e no sistema nervoso. As classes terapêuticas mais frequentes em uso pelos entrevistados foram os medicamentos utilizados para o DM, os agentes que atuam no sistema renina angiotensina, os diuréticos, os agentes modificadores de lipídeos e os agentes trombolíticos. Os medicamentos mais frequentes em uso foram metformina, losartan, glibenclamida e sinvastatina (Tabela 1). Esses resultados correspondem as comorbidades mais prevalentes observadas no estudo, que foram a hipertensão, com $76 \%$, a dislipidemia, com $44 \%$, e a obesidade, com 32\%. Além disso, 31\% relataram que têm ou já tiveram depressão e observou-se uma participação importante de antidepressivos entre os agentes que atuam no sistema nervoso.

Dentre os entrevistados, 56,5\% - margem de erro no IC95\% igual a 3,4\% - estavam em polifarmácia. O grupo de polifarmácia apresentou maior frequência de pessoas do sexo feminino, com idade mais avançada e maior tempo de diagnóstico em comparação com o grupo sem 


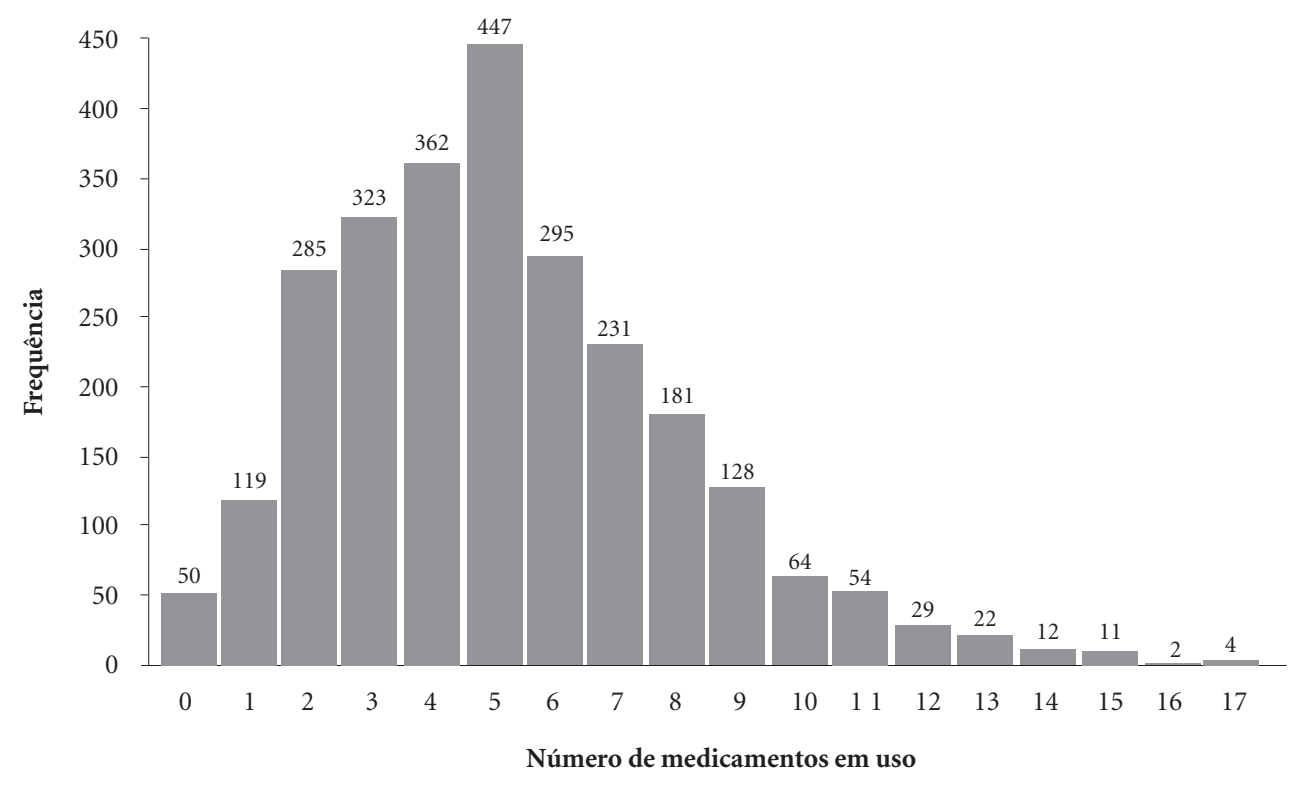

Gráfico 1. Histograma da distribuição de frequência da quantidade de medicamentos em uso pelos entrevistados.

polifarmácia. Verificou-se ainda maior número de comorbidades associadas ao DM, de consultas médicas no último ano, de interrupção das atividades habituais e menor prática regular de atividade física e pior autopercepção de saúde no grupo de polifarmácia. Todas as variáveis mostraram associação estatística significante com a polifarmácia na análise bivariada (Tabela 2).

A análise de regressão logística indicou que os fatores associados positiva e significantemente à ocorrência de polifarmácia em pacientes DM são idade acima de 40 anos, autopercepção de saúde ruim ou muito ruim, presença de cinco ou mais comorbidades, tempo médio de diagnóstico acima de 10 anos, ter consultado ao médico quatro vezes ou mais no último ano, ausência de atividade física regular, interrupção das atividades habituais nos últimos 15 dias e ter plano de saúde particular (Tabela 3).

\section{Discussão}

A prevalência de polifarmácia encontrada neste estudo, considerando-se o uso de cinco ou mais medicamentos, foi de $56,5 \%$. Conforme a literatura a ocorrência de polifarmácia em pacientes com DM no Brasil varia de $26,7 \%$ a $46,3 \%$, pro- porções inferiores à encontrada no presente estu$\mathrm{do}^{14,15,22}$. Estudo indiano relata uma prevalência de polifarmácia em indivíduos com DM tipo 2 de 25,5\% em pacientes não idosos e de 41,6\% em pacientes idosos ${ }^{23}$. Qato et al. ${ }^{10}$ demonstraram um aumento significativo da prevalência de polifarmácia entre idosos em um intervalo de cinco anos, associado principalmente ao aumento do consumo de suplementos alimentares e das múltiplas prescrições de medicamentos em pacientes com comorbidades.

Outro aspecto relacionado ao aumento da polifarmácia decorre do fato de que indivíduos com DM são mais propensos a estarem em situação de polifarmácia ${ }^{7,12}$. Isso pode ser parcialmente explicado pelo aumento da dose dos medicamentos em uso ou da prescrição de novos medicamentos quando não se obtém a adesão ao tratamento proposto e o controle adequado do DM e das comorbidades, o que pode levar a maior risco de interações, maior dificuldade na adesão e controle das doenças ${ }^{24,25}$.

Os medicamentos com ação no sistema cardiovascular foram os mais utilizados, seguidos daqueles com ação no trato alimentar e metabolismo e sistema nervoso, conforme observado no estudo de Pereira et al..$^{20} \mathrm{em}$ pacientes hipertensos e diabéticos e por Baldoni et al. ${ }^{26}$ em idosos. Bauer e 
Tabela 1. Distribuição das especialidades farmacêuticas por grupos e subgrupos, segundo a Classificação Anatômica Terapêutica (ATC).

\begin{tabular}{|c|c|c|c|c|c|}
\hline Nível I $(\mathrm{N}=13629)^{\mathrm{a}}$ & $\%$ & Nível II $(\mathrm{n}=2619)^{\mathrm{b}}$ & $\%$ & Nível V $(\mathbf{n}=2619)^{\mathrm{b}}$ & $\%$ \\
\hline \multirow[t]{7}{*}{ Cardiovascular (C) } & 40,0 & $\begin{array}{l}\text { Agentes que atuam no sistema } \\
\text { renina-angiotensina (C09) }\end{array}$ & 56,1 & Losartan (C09CA01) & 32,1 \\
\hline & & Diuréticos (C03) & 39,4 & Sinvastatina (C09CA01) & 29,2 \\
\hline & & Agentes modificadores de lipídeos (C10) & 32,6 & $\begin{array}{l}\text { Hidroclorotiazida } \\
\text { (C03AA03) }\end{array}$ & 23,9 \\
\hline & & Betabloqueadores (C07) & 23,8 & Enalapril (C09AA02) & 15,2 \\
\hline & & Bloqueadores dos canais de cálcio (C08) & 18,7 & Anlodipino (C08CA01) & 14,4 \\
\hline & & Terapia cardíaca (C01) & 6,3 & Furosemida (C03CA01) & 13,3 \\
\hline & & Outros ${ }^{\star *}$ & 6,8 & Atenolol (C07AB03) & 11,1 \\
\hline \multirow{6}{*}{$\begin{array}{l}\text { Trato Alimentar e } \\
\text { Metabolismo (A) }\end{array}$} & 35,4 & Medicamentos utilizados no diabetes (A10) & 94,5 & Metformina (A10BA02) & 68,6 \\
\hline & & Antidiabéticos orais (A10B) & 84,5 & Glibenclamida (A10BB01) & 31,5 \\
\hline & & Insulinas e análogos (A10A) & 30,2 & Insulina NPH (A10AC01) & 27,1 \\
\hline & & $\begin{array}{l}\text { Medicamentos para transtornos } \\
\text { relacionados à acidez (A02) }\end{array}$ & 18,7 & Omeprazol (A02BC01) & 16,0 \\
\hline & & Vitaminas (A11) & 6,9 & Glimepirida (A10BB12) & 5,7 \\
\hline & & Outros ${ }^{* *}$ & 4,5 & Gliclazida (A10BB09) & 5,6 \\
\hline \multirow[t]{5}{*}{ Nervoso $(\mathrm{N})$} & 10,2 & Psicoanalépticos (N06) & 16,5 & Clonazepam (N03AE01) & 6,6 \\
\hline & & Antiepilépticos (N03) & 11,1 & Fluoxetina (N06AB03) & 4,6 \\
\hline & & Psicolépticos (N05) & 9,9 & Amitriptilina (N06AA09) & 3,7 \\
\hline & & Analgésicos (N02) & 5,4 & Diazepam (N05BA01) & 2,6 \\
\hline & & Outros ${ }^{\star *}$ & 3,4 & Sertralina (N06AB06) & 2,3 \\
\hline \multirow{2}{*}{$\begin{array}{c}\text { Sangue e orgãos } \\
\text { hematopoiéticos (B) }\end{array}$} & 6,9 & Agentes antitrombóticos (B01) & 30,4 & Ácido Acetilsalicílico & 27,3 \\
\hline & & Outros ${ }^{* *}$ & 2,4 & (B01AC06) & \\
\hline \multirow{2}{*}{$\begin{array}{l}\text { Hormônios, excluíndo } \\
\text { insulinas }(\mathrm{H})\end{array}$} & 2,1 & Terapia da tireóide (H03) & 8,8 & Levotiroxina (H03AA01) & 8,6 \\
\hline & & Outros ${ }^{\star *}$ & 3,1 & & \\
\hline \multirow{3}{*}{$\begin{array}{l}\text { Músculo-Esquelético } \\
\text { (M) }\end{array}$} & 2,0 & Antiinflamatórios e antirreumáticos (M01) & 5,0 & Alopurinol (M04AA01) & 1,9 \\
\hline & & Preparações antigota (M04) & 2,0 & Alendronato (M05BA04) & 1,5 \\
\hline & & Outros ${ }^{\star *}$ & 2,3 & Ibuprofeno (M01AE01) & 1,4 \\
\hline \multirow[t]{3}{*}{ Respiratório (R) } & 1,3 & $\begin{array}{l}\text { Agentes contra doenças obstrutivas das } \\
\text { vias aéreas (R03) }\end{array}$ & 2,6 & $\begin{array}{l}\text { Formoterol + Budesonida } \\
(\text { R03AK07) }\end{array}$ & 1,4 \\
\hline & & Anti-histamínicos para uso sistêmico (R06) & 1,8 & & \\
\hline & & Outros ${ }^{* *}$ & 0,6 & & \\
\hline Outros* & 2,2 & & & & \\
\hline
\end{tabular}

${ }^{\mathrm{a}}=$ Total de medicamentos em uso pelos entrevistados; ${ }^{\mathrm{b}}=$ Total de entrevistados. ${ }^{*}$ Soma dos medicamentos em uso de códigos ATC Nível I não apresentados separadamente (D, G, J, L, P, S e V), por apresentarem menor frequência de uso. ${ }^{* \star}$ Soma dos medicamentos em uso de códigos ATC Nível II não apresentados separadamente (exemplo: C02, C04, C05 para ATC Nível I = C), por apresentarem menor frequência de uso.

Nauck $^{19}$ identificaram que os medicamentos mais utilizados pelos pacientes DM, em um centro especializado no tratamento do DM na Alemanha, foram para o trato alimentar e metabolismo, seguido pelos utilizados no sistema cardiovascular e sistema nervoso. No estudo de Guidoni et al. ${ }^{27}$, os medicamentos mais utilizados por pacientes com DM foram para o sistema cardiovascular, sistema nervoso, sangue e órgãos hematopoiéticos e trato alimentar e metabolismo. Em outros estudos com pacientes idosos, os mais utilizados foram para o sistema cardiovascular, nervoso e trato alimentar e metabolismo ${ }^{14,15}$. Em geral, a semelhança entre os medicamentos utilizados se deve às comorbidades mais prevalentes associadas ao DM, hipertensão, dislipidemia e depressão, também observadas no presente estudo ${ }^{2,28}$.

Segundo a $\operatorname{ADA}(2016)^{28,29}$, hipertensão e dislipidemia são comumente encontradas em pacientes com DM, que apresentam também maior prevalência de depressão do que as pessoas sem $\mathrm{DM}$, o que pode dificultar a gestão do tratamento dos pacientes. Há fortes evidências de que a depressão em pessoas com diabetes aumenta o risco 
Tabela 2. Associação bivariada das variáveis sociodemográficas e clínicas com a polifarmácia em pacientes com DM em Minas Gerais.

\begin{tabular}{|c|c|c|c|c|}
\hline \multirow{2}{*}{ Variáveis } & \multirow{2}{*}{$\begin{array}{c}\text { Sem polifarmácia } \\
\mathbf{n}(\%)\end{array}$} & \multirow{2}{*}{$\begin{array}{c}\text { Polifarmácia } \\
\text { n (\%) }\end{array}$} & \multicolumn{2}{|c|}{ Total } \\
\hline & & & $\mathbf{N}$ & $\%^{*}$ \\
\hline Sexo & & & \multicolumn{2}{|c|}{$\mathrm{p}<0,001$} \\
\hline Masculino & $415(51,2)$ & $398(48,8)$ & 811 & 31,0 \\
\hline Feminino & $724(40,0)$ & $1084(60,0)$ & 1808 & 69,0 \\
\hline Estado Civil & & & \multicolumn{2}{|c|}{$\mathrm{p}<0,001$} \\
\hline Solteiro & $229(57,7)$ & $168(42,3)$ & 397 & 15,2 \\
\hline Casado & $583(44,6)$ & $725(55,4)$ & 1308 & 49,9 \\
\hline Viúvos & $208(32,0)$ & $443(68,0)$ & 651 & 24,9 \\
\hline Outros & $119(45,2)$ & $144(54,8)$ & 263 & 10,0 \\
\hline Escolaridade & & & \multicolumn{2}{|c|}{$\mathrm{p}<0,001$} \\
\hline$<4$ anos & $420(37,2)$ & $708(62,8)$ & 1128 & 43,1 \\
\hline 4 anos ou mais & $719(48,2)$ & $772(51,8)$ & 1491 & 56,9 \\
\hline Idade & & & \multicolumn{2}{|c|}{$\mathrm{p}<0,001$} \\
\hline$<40$ anos & $188(78,7)$ & $51(21,3)$ & 239 & 9,1 \\
\hline 40 a 59 anos & $408(52,1)$ & $375(47,9)$ & 783 & 29,9 \\
\hline 60 ou mais & $543(34,0)$ & $1054(66,0)$ & 1597 & 61,0 \\
\hline Raça ou cor & & & \multicolumn{2}{|c|}{$p=0,069$} \\
\hline Branco & $470(41,2)$ & $671(58,8)$ & 1141 & 43,6 \\
\hline Pardo & $477(44,4)$ & $598(55,6)$ & 1075 & 41,0 \\
\hline Negro & $175(48,9)$ & $183(51,1)$ & 358 & 13,7 \\
\hline Outros & $14(41,2)$ & $20(58,8)$ & 34 & 1,3 \\
\hline Autopercepção de saúde & & & \multicolumn{2}{|c|}{$\mathrm{p}<0,001$} \\
\hline Muito bom/bom & $539(54,8)$ & $445(45,2)$ & 984 & 37,6 \\
\hline Regular & $498(40,5)$ & $733(59,5)$ & 1231 & 47,0 \\
\hline Ruim/Muito ruim & $100(24,9)$ & $302(75,1)$ & 402 & 15,3 \\
\hline Tipo de DM & & & \multicolumn{2}{|c|}{$\mathrm{p}=0,123$} \\
\hline I & $130(47,8)$ & $142(52,2)$ & 272 & 10,4 \\
\hline II & $940(42,9)$ & $1252(57,1)$ & 2192 & 83,7 \\
\hline Diagnóstico de DM & & & \multicolumn{2}{|c|}{$\mathrm{p}<0,001$} \\
\hline$<10$ anos & $750(51,9)$ & $694(48,1)$ & 1444 & 55,1 \\
\hline 10 anos ou mais & $389(33,1)$ & $786(66,9)$ & 1175 & 44,9 \\
\hline Comorbidades & & & \multicolumn{2}{|c|}{$\mathrm{p}<0,001$} \\
\hline$<5$ & $841(61,2)$ & $533(38,8)$ & 1374 & 52,5 \\
\hline 5 ou mais & $298(23,9)$ & $947(76,1)$ & 1245 & 47,5 \\
\hline Consultas médicas & & & \multicolumn{2}{|c|}{$\mathrm{p}<0,001$} \\
\hline$<3$ & $601(57,1)$ & $451(42,9)$ & 1052 & 40,2 \\
\hline 3 ou mais & $538(34,3)$ & $1029(65,7)$ & 1567 & 59,8 \\
\hline Plano de saúde privado & & & \multicolumn{2}{|c|}{$\mathrm{p}<0,001$} \\
\hline Sim & $308(38,6)$ & $490(61,4)$ & 798 & 30,5 \\
\hline Não & $831(45,6)$ & $990(54,4)$ & 1821 & 69,5 \\
\hline Faz atividade física regular & & & \multicolumn{2}{|c|}{$\mathbf{p}<\mathbf{0 , 0 0 1}$} \\
\hline Sim & $559(51,8)$ & $521(48,2)$ & 1080 & 41,2 \\
\hline Não & $579(37,6)$ & $959(62,4)$ & 1538 & 58,7 \\
\hline Deixou de realizar atividac & iltimos 15 dias & & \multicolumn{2}{|c|}{$\mathrm{p}<0,001$} \\
\hline Sim & $198(28,8)$ & $489(71,2)$ & 687 & 26,2 \\
\hline Não & $941(48,7)$ & $991(51,3)$ & 1932 & 73,8 \\
\hline
\end{tabular}

Sem polifarmácia: 0 a 4 medicamentos em uso; Polifarmácia: $\geq 5$ medicamentos em uso. (n) Número de entrevistados por variável dentro das categorias polifarmácia ou sem polifarmácia. (\%) Porcentagem de variável dentro das categorias polifarmácia ou sem polifarmácia. (N) Número de entrevistados por variável em relação ao total de entrevistados. $\left(\%{ }^{*}\right)$ Porcentagem da variável em relação ao total de entrevistados. Sem informação não foi considerado na análise. Valor de p: $<0,20$. 
Tabela 3. Fatores associados à polifarmácia em pacientes com DM em Minas Gerais.

\begin{tabular}{|c|c|c|c|}
\hline \multirow{2}{*}{ Variáveis } & \multicolumn{3}{|c|}{ Polifarmácia } \\
\hline & OR & \multicolumn{2}{|c|}{ IC95\% } \\
\hline \multicolumn{4}{|l|}{ Idade } \\
\hline$<40$ anos & 1,00 & & \\
\hline 40 a 59 anos & 2,46 & 1,68 & 3,61 \\
\hline 60 a 69 anos & 4,58 & 3,18 & 6,60 \\
\hline \multicolumn{4}{|l|}{ Autopercepção de saúde } \\
\hline Muito bom/Bom & 1,00 & & \\
\hline Regular* & 1,12 & 0,92 & 1,37 \\
\hline Ruim/Muito ruim & 1,73 & 1,26 & 2,38 \\
\hline \multicolumn{4}{|l|}{ Comorbidades } \\
\hline$<5$ & 1,00 & & \\
\hline 5 ou mais & 3,45 & 2,84 & 4,19 \\
\hline \multicolumn{4}{|l|}{ Diagnóstico de DM } \\
\hline$<10$ anos & 1,00 & & \\
\hline 10 anos ou mais & 1,64 & 1,36 & 1,98 \\
\hline \multicolumn{4}{|c|}{ Consultas médicas no último ano } \\
\hline$<4$ & 1,00 & & \\
\hline 4 ou mais & 1,79 & 1,48 & 2,16 \\
\hline \multicolumn{4}{|l|}{ Atividade Física regular } \\
\hline $\operatorname{Sim}$ & 1,00 & & \\
\hline Não & 1,47 & 1,22 & 1,78 \\
\hline \multicolumn{4}{|c|}{$\begin{array}{l}\text { Deixou de realizar atividade habitual nos últimos } \\
15 \text { dias }\end{array}$} \\
\hline Não & 1,00 & & \\
\hline Sim & 1,30 & 1,03 & 1,64 \\
\hline \multicolumn{4}{|l|}{ Plano de saúde privado } \\
\hline Não & 1,00 & & \\
\hline Sim & 1,39 & 1,13 & 1,70 \\
\hline
\end{tabular}

OR: Razão de chances, do inglês Odds Ratio.

* Não significante. Valor de p: $<0,05$.

de complicações a ele relacionadas. A depressão tem sido associada à hipoglicemia, a complicações relacionadas com o DM, bem como à percepção das limitações funcionais decorrentes do $\mathrm{DM}^{2,29}$.

As classes terapêuticas mais frequentes foram os medicamentos utilizados no diabetes, agentes que atuam no sistema renina angiotensina, os diuréticos e os agentes modificadores de lipídeos, corroborado pelos estudos de Guidoni et al. ${ }^{27} \mathrm{e}$ Bauer e Nauck ${ }^{19}$. O tratamento da hipertensão em pacientes com DM preconizado pela ADA (2016) e pelas IV Diretrizes Brasileiras de Hipertensão (2010) deve incluir inibidor da enzima conversora da angiotensina ou antagonista do receptor de angiotensina e, em caso de impossibilidade do uso desses agentes, o uso de diuréticos, o que foi observado no presente estudo ${ }^{28,30}$.
Dentre os medicamentos mais utilizados conforme a classificação ATC, apenas a glimepirida e a sertralina não são disponibilizadas pelo Sistema Único de Saúde (SUS) ${ }^{31,32}$. Além disso, quase dois terços dos medicamentos foram adquiridos por meio de farmácias públicas. Tal resultado indica participação importante do SUS no acesso a medicamentos em Minas Gerais, no período da pesquisa. Contribuiu para isso a implementação da Rede Farmácia de Minas, inserida no Programa Estadual de Reestruturação da Assistência Farmacêutica, como uma estratégia para ampliar o acesso e o uso racional dos medicamentos no âmbito do SUS ${ }^{33}$.

Não foram encontrados estudos em pacientes com DM que associem a polifarmácia com suas variáveis explicativas. Somente estudos em idosos investigaram a quantidade de medicamentos utilizados com seus fatores associados. Nesse contexto, verifica-se que a população deste estudo apresenta características semelhantes à população idosa, com idade média de 61,3 anos e mais de $60 \%$ dos entrevistados declarando ter 60 anos ou mais. Assim, estudos realizados com população de idosos foram utilizados para comparação.

Em relação à idade, quanto maior a faixa etária dos pacientes com DM, maior a chance de o paciente apresentar polifarmácia, o que está de acordo com os resultados de outros estudos ${ }^{11,19,34,35}$. A idade média dos pacientes foi semelhante à encontrada nos estudos de Araújo et al. ${ }^{22}$ e Bauer e Nauck ${ }^{19}$ em pacientes com DM de Fortaleza, Brasil e de Bad Lauterberg im Harz, na Alemanha.

Outro fator que contribui para o aumento do número de medicamentos é a maior quantidade de comorbidades ${ }^{7,24,35,36}$. Neste ponto, observa-se que muitos indivíduos não conseguem obter um controle adequado de suas condições de saúde. No estudo de Gomes et al. ${ }^{36}$, realizado em pacientes com DM que apresentavam outras comorbidades, menos de $30 \%$ dos pacientes atingiram a meta de controle da pressão arterial, cerca de $25 \%$ atingiram o índice de IMC $<25 \mathrm{Kg} / \mathrm{m}^{2}, 21 \%$ conseguiram o controle do nível de LDL-Colesterol e $46 \%$ conseguiram o controle adequado de hemoglobina glicada. A presença de um maior número de comorbidades, associado a um controle inadequado dessas condições de saúde contribuem muito para o aumento do número de medicamentos em uso.

Ter consultado o médico quatro ou mais vezes no último ano está associado com polifarmácia em pacientes com DM, o que também foi constatado em outros estudos com pacientes 
idosos ${ }^{35,36}$. Linjakumpu et al. ${ }^{37}$ apontam que o aumento da utilização de serviços de saúde pelos idosos de maior idade pode ser responsável pelo maior uso de medicamentos. Ressalta-se aqui a importância da educação médica continuada, da observância dos protocolos clínicos e das diretrizes terapêuticas, da qualidade da prescrição médica e da orientação farmacêutica para contribuir para o uso racional do número elevado de medicamentos em uso pelos pacientes com DM. Isso demonstra a importância de um cuidado multidisciplinar em saúde que envolve ações de educação em saúde, autocuidado, adesão às medidas propostas, intervenções não medicamentosas, dentre outras ${ }^{37-40}$.

O tempo de diagnóstico médio acima de 10 anos mostrou associação com a polifarmácia. De acordo com o Caderno de Atenção Básica - Diabetes $^{41}$ e Lipska et al. ${ }^{42}$, com o aumento do tempo de diagnóstico observa-se o aparecimento de complicações microvasculares e macrovasculares nos pacientes, principalmente quando esses desconheciam ter a doença na época do diagnóstico. Além disso, observa-se que em nove anos 75\% dos pacientes com DM necessitam utilizar mais de um medicamento para o controle glicêmico. Ambos os fatores incrementam o número de medicamentos em uso e favorecem a presença de polifarmácia ${ }^{43}$.

A autopercepção de saúde ruim ou muito ruim mostrou associação com a polifarmácia, aspecto observado também em outros estudos ${ }^{11,44}$. Segundo Santos et al. ${ }^{44}$, os pacientes que apresentam uma pior autopercepção de saúde buscam nos medicamentos uma solução para esses problemas. Em um estudo conduzido no Canadá ${ }^{45}$, observou-se um maior relato de autopercepção de saúde ruim ou muito ruim em relação ao observado no nosso, associado principalmente à presença de depressão e deficiências associadas ou decorrentes de DM, além de outras comorbidades crônicas.

A ausência de atividade física regular e o deixar de realizar atividades habituais são fatores associados à polifarmácia em pacientes com DM tipo 2. Segundo a Sociedade Brasileira de Diabetes $^{2}$, a atividade física, associada ao plano alimentar, pode ser benéfica para auxiliar no controle glicêmico, na perda ponderal e no aumento da massa muscular, o que deve colaborar para a redução da resistência à insulina e melhor controle da doença. Além disso, Silva et al ${ }^{46}$ relataram que a interrupção das atividades habituais está relacionada ao uso de maior número de medicamentos.
Observou-se que ter plano de saúde esteve associado à polifarmácia como relatado por outros estudos ${ }^{11,46}$. Segundo Duarte et al. ${ }^{47}$, pessoas que têm plano de saúde possuem uma maior renda e acesso mais facilitado aos profissionais de saúde e aos medicamentos disponíveis no mercado. Em estudo conduzido em pacientes com DM vinculados ao Medicare, nos Estados Unidos, observou-se que metade dos indivíduos com controle glicêmico inadequado não teve seu tratamento intensificado ao longo de cinco anos, o que leva a complicações do DM e contribui para o aumento da polifarmácia ${ }^{48}$.

As limitações deste estudo estão relacionadas à utilização de um período recordatório de 15 dias para a avaliação do uso de medicamentos, $\mathrm{o}$ que pode gerar viés de memória. Tentou-se, contudo, minimizar esse viés através da comprovação de uso por meio da apresentação das embalagens dos produtos e prescrições. Além disso, o método transversal não permite o estabelecimento de relações de causa e efeito. A coleta de dados foi realizada em horário habitual de trabalho das pessoas, das 08 às 18 horas, o que pode ter concorrido para a seleção de amostra de idade mais avançada, de pessoas laboralmente inativas. Sugere-se que novos estudos devam avaliar os possíveis benefícios e malefícios da polifarmácia, da associação medicamentosa e da influência destas na qualidade de vida dos pacientes com DM, com o objetivo de alcançar o uso racional de medicamentos e otimizar o cuidado a esses pacientes.

A polifarmácia apresenta uma relevante prevalência entre os pacientes com DM nos municípios de Minas Gerais. A maioria dos entrevistados estava em uso de polifarmácia, o que aumenta o risco de reações adversas, a toxicidade cumulativa e as interações medicamentosas. O presente estudo propiciou um conhecimento mais aprofundado dos fatores relacionados à polifarmácia e espera-se que estas informações possam contribuir para a otimização do cuidado aos pacientes com essa condição de saúde, reforçar a necessidade de ações de educação em saúde e da busca pelo uso racional dos medicamentos. Fatores como o envelhecimento da população, o aumento de comorbidades e o acesso aos serviços de saúde contribuem para o aumento do número de medicamentos utilizados pela população com DM. Por isso, há necessidade de se disponibilizar um número suficiente de profissionais capacitados para prover o cuidado adequado, melhorar a qualidade do uso de medicamentos e minimizar as consequências negativas na saúde dessa população. 


\section{Colaboradores}

MRR Silva participou do planejamento e da coleta dos dados, análise dos dados e redação do artigo. LM Diniz colaborou na elaboração do projeto, planejamento da coleta de dados, análise dos resultados e revisão crítica do artigo. JBR Santos colaborou na análise dos dados e redação do artigo. EA Reis colaborou na análise dos dados e revisão crítica do artigo. AR Mata e VE Araújo colaboraram no planejamento e supervisão da coleta dos dados e revisão crítica do artigo. J Álvares colaborou na elaboração do projeto, planejamento da coleta dos dados e revisão crítica do artigo. FA Acurcio colaborou na elaboração e orientação do projeto, planejamento da coleta de dados, análise dos resultados e revisão crítica do artigo. Todos os autores aprovaram a versão a ser publicada.

\section{Agradecimentos}

Os autores agradecem o apoio do Conselho $\mathrm{Na}$ cional de Desenvolvimento Científico e Tecnológico, da Fundação de Amparo a Pesquisa de Minas Gerais, do Grupo de Pesquisas em Farmacoepidemiologia da UFMG e da Secretaria de Estado da Saúde de Minas Gerais.

Os autores declaram não possuir conflitos de interesses.

\section{Referências}

1. American Diabetes Association. Standart of Medical Care in Diabetes. Introduction. Diabetes Care 2016; 39(Supl. 1):S1-S2.

2. Sociedade Brasileira de Diabetes. Diretrizes da Sociedade Brasileira de Diabetes. 2014-2015. São Paulo: AC Farmacêutica; 2015.

3. Wild S, Roglic G, Green A, Sicree R, King H. Global prevalence of diabetes: estimates for the year 2000 and projections for 2030. Diabetes Care 2004; 27(5):10471053.

4. International Diabetes Federation. Diabetes atlas. $6^{\text {th }}$ ed. 2014 Update. [acessado 2015 Mar 11]. Disponível em: http://www.idf.org/sites/default/files/Atlas-poster-2014_EN.pdf

5. International Diabetes Federation. Diabetes atlas. $6^{\text {th }}$ ed. 2014 Update. [acessado 2015 Mar 11]. Disponível em: http://www.idf.org/sites/default/files/6th-Edition -Estimates_Update_2014.xls

6. American Diabetes Association (ADA). 11. Children and Adolescents. Diabetes Care 2016 Jan; 39(Supl. 1):S86-S93.

7. American Diabetes Association (ADA). 10. Older Adults. Diabetes Care 2016; 39(Supl. 1):S81-S85.

8. Mata AR, Álvares J, Diniz LM, Silva MRR, Santos BRA, Guerra Júnior AA, Cherchiglia ML, Andrade EI, Godman B, Acurcio FA. Quality of life of patients with Diabetes Melito Types 1 and 2 from a referal health centre in Minas Gerais, Brazil. Expert Rev Clin Pharmacol 2016; 9(5):739-746.

9. Freeman JS, Gross B. Potential drug interactions associated with treatments for type 2 diabetes and its comorbidities: a clinical pharmacology review. Expert Rev Clin Pharmacol 2012; 5(1):31-42.

10. Qato DM, Wilder J, Schumm LP, Gillet V, Alexander GC. Changes in Prescription and Over-the-Counter Medication and Dietary Supplement Use Among Older Adults in the United States, 2005 vs 2011. JAMA Intern Med 2016; 176(4):473-482.
11. Carvalho MF, Romano-Lieber NS, Bergsten-Mendes G Secoli SR, Ribeiro E, Lebrão ML, Duarte YA. Polypharmacy among the elderly in the city of São Paulo, Brazil - SABE Study. Rev Bras Epidemiol 2012; 15(4):817-827.

12. Viktil KK, Blix HS, Moger TA, Reikvam A. Polypharmacy as commonly defined is an indicator of limited value in the assessment of drug-related problems. $\mathrm{Br} \mathrm{J}$ Clin Pharmacol 2007; 63(2):187-195.

13. Markovi-Pekovi V, Škrbi R, Petrovi A, Vlahovi -Pal evski V, Mrak J, Bennie M, Fadare J, Kwon HY, Schiffers K, Truter I, Godman B. Polypharmacy among the elderly in the Republic of Srpska: extent and implications for the future. Expert Rev Pharmacoecon Outcomes Res 2016; 16(5):609-618.

14. Vitoi NC, Fogal AS, Nascimento CM, Franceschini SD, Ribeiro AQ. Prevalence and associated factors of diabetes in the elderly population in Viçosa, Minas Gerais, Brazil. Rev Bras Epidemiol 2015; 18(4):953-965

15. Moraes SA, Freitas IC, Gimeno SG, Mondini L. Diabetes melito prevalence and associated factors in adults in Ribeirão Preto, São Paulo, Brazil, 2006: OBEDIARP Project. Cad Saude Publica 2010; 26(5):929-941.

16. Sharma M, Nazareth I, Petersen I. Trends in incidence, prevalence and prescribing in type 2 diabetes melito between 2000 and 2013 in primary care: a retrospective cohort study. BMJ Open. 2016; 6(1):e010210. Erratum in: BMJ Open 2016; 6(5):e010210corr 1.

17. Andersson T, Ahlbom A, Carlsson S. Diabetes Prevalence in Sweden at Present and Projections for Year 2050. PLoS One 2015; 10(11):e0143084.

18. Meo SA. Prevalence and future prediction of type 2 diabetes melito in the Kingdom of Saudi Arabia: A systematic review of published studies. J Pak Med Assoc 2016; 66(6):722-725.

19. Bauer S, Nauck MA. Polypharmacy in people with Type 1 and Type 2 diabetes is justified by current guidelines - a comprehensive assessment of drug prescriptions in patients needing inpatient treatment for diabetes-associated problems. Diabet Med 2014; 31(9):1078-1085. 
20. Pereira VO, Acurcio FA, Guerra Júnior AA, Silva GD, Cherchiglia ML. Use of medicines by individuals with hypertension and diabetes in municipalities covered by the Pharmacy Network in Minas Gerais State, Brazil. Cad Saude Publica 2012; 28(8):1546-1558.

21. Instituto Brasileiro de Geografia e Estatística (IBGE). Estimativas de população para $1^{\circ}$ de julho de 2013. Rio de Janeiro; IBGE; 2013. [acessado 2015 Ago 14]. Disponível em: http://www.ibge.gov.br/home/estatistica/ populacao/estimativa2013/estimativa_tcu.shtm

22. Araújo MF, Santos Alves PJ, Veras VS, Araújo TM, Zanetti ML, Damasceno MM. Drug interactions in Brazilian type 2 diabetes patients. Int J Nurs Pract 2013; 19(4):423-430.

23. Shastry R, Prabha Adhikari MR, Sheetal U, Kotian S. Comparison of polypharmacy between geriatric and nongeriatric diabetic patients. Int J Pharm 2016; 6(1):85-87.

24. Pereira LRL, Andrade RCG, Pereira JGC, Marchetti JM. Avaliação de prescrições de medicamentos para pacientes com Diabetes Melito atendidos por uma Unidade Básica de saúde. Rev. Ciênc. Farm. Básica Apl. 2005; 26(3):199-203.

25. Jarab AS, Almrayat R, Alqudah S, Thehairat E, Mukattash TL, Khdour M, Pinto S. Predictors of non-adherence to pharmacotherapy in patients with type 2 diabetes. Int J Clin Pharm 2014; 36(4):725-733.

26. Baldoni AO, Ayres LR, Martinez EZ, Dewulf NLS, Santos V, Obreli-Neto PR, Pereira RLR. Pharmacoepidemiological profile and polypharmacy indicators in elderly outpatients. Braz. J. Pharm. Sci. 2013; 49(3):443-452.

27. Guidoni CM, Borges AP, Freitas O, Pereira LR. Analysis of treatment of comorbidities and the profile of medical consultations for diabetes melito. Cien Saude Colet 2013; 18(10):3015-3022.

28. American Diabetes Association (ADA). 8. Cardiovascular Disease and Risk Management. Diabetes Care 2016; 39(Supl. 1):S60-S71.

29. American Diabetes Association (ADA). 3. Foundations of Care and Comprehensive Medical Evaluation. Diabetes Care 2016; 39(Supl. 1):S23-S35.

30. Sociedade Brasileira de Cardiologia. VI Diretrizes Brasileiras de Hipertensão. Arq Bras Cardiol 2010; 95(1):151.

31. Minas Gerais. Secretaria de Estado de Saúde de Minas Gerais (SESMG). Componentes Básico e Estratégico da Assistência Farmacêutica. $2^{\text {a }}$ ed. Belo Horizonte: SESMG; 2012.

32. Brasil. Ministério da Saúde (MS). Relação Nacional de Medicamentos Essenciais: RENAME 2014. 9a ed. rev. e atual. Brasília: MS; 2015.

33. Minas Gerais. Rede Farmácia de Minas. Plano Estadual de Estruturação da Rede de Assistência Farmacêutica: Uma estratégia para ampliar o acesso e o uso racional de medicamentos no SUS. [acessado 2016 Jul 19]. Disponível em: https://www.saude.mg.gov.br/images/documentos/02\%20Farmacia_de_Minas_050608.pdf

34. Jyrkkä J, Enlund H, Korhonen MJ, Sulkava R, Hartikainen S. Patterns of druguse and factors associated with polypharmacy and excessive polypharmacy in elderly persons: results of the Kuopio 75+ study: a cross-sectional analysis. Drugs Aging 2009; 26(6):493-503.

35. Loyola Filho AI, Uchoa E, Lima-Costa MF. A population-based study on use of medication by the elderly in Greater Metropolitan Belo Horizonte, Minas Gerais, Brazil. Cad Saude Publica 2006; 22(12):2657-2667.
36. Gomes MB, Gianella D, Faria M, Tambascia M, Fonseca RM, Réa R, Macedo G, Modesto Filho J, Schmid H, Bittencourt AV, Cavalcanti S, Rassi N, Pedrosa H, Atala Dib S. Prevalence of Type 2 diabetic patients within the targets of care guidelines in daily clinical practice: a multi-center study in Brazil. Rev Diabet Stud 2006; $3(2): 82-87$

37. Linjakumpu T, Hartikainen S, Klaukka T, Veijola J, Kivelä SL, Isoaho R. Use of medications and polypharmacy are increasing among the elderly. J Clin Epidemiol 2002; 55(8):809-817.

38. Cardoso AF, Queirós P, Ribeiro CF. Therapeutic self care management interventions for individuals with diabetes melito: systematic review. Rev Port Saúde Pública 2015; 33(2):246-255.

39. Costa JA, Balga RS, Alfenas RC, Cotta RM. Health promotion and diabetes: discussing the adherence and motivation of diabetics that participate in health programs. Cien Saude Colet 2011; 16(3):2001-2009.

40. Torres HC, Souza ER, Lima MHM, Bodstein RC. Intervenção educativa para o autocuidado de indíviduos com diabetes melito. Acta paul. enferm. 2011; 24(4):514-519.

41. Brasil. Ministério da Saúde (MS). Estratégias para o cuidado da pessoa com doença crônica: diabetes melito. Brasília: MS; 2013. (Cadernos de Atenção Básica, nº 36).

42. Lipska KJ, Krumholz H, Soones T, Lee SJ. Polypharmacy in the Aging Patient: A Review of Glycemic Control in Older Adults With Type 2 Diabetes. JAMA 2016; 315(10):1034-1045.

43. Polonsky WH, Fisher L, Schikman CH, Hinnen DA, Parkin CG, Jelsovsky Z, Petersen B, Schweitzer M, Wagner RS. Structured self-monitoring of blood glucose significantly reduces A1C levels in poorly controlled, noninsulin-treated type 2 diabetes: results from the Structured Testing Program study. Diabetes Care 2011; 34(2):262-267.

44. Santos TR, Lima DM, Nakatani AY, Pereira LV, Leal GS, Amaral RG. Medicine use by the elderly in Goiania, Midwestern Brazil. Rev Saude Publica 2013; 47(1):94103.

45. Badawi G, Gariépy G, Pagé V, Schmitz N. Indicators of self-rated health in the Canadian population with diabetes. Diabet Med 2012; 29(8):1021-1028.

46. Silva AL, Ribeiro AQ, Klein $\mathrm{CH}$, Acurcio FA. Use of medications by elderly Brazilians according to age: a postal survey. Cad Saude Publica 2012; 28(6):10331045.

47. Duarte LR, Gianini RJ, Ferreira LR, Camargo MAS, Galhardo SD. Hábitos de consumo de medicamentos entre idosos usuários do SUS e de plano de saúde. Cad Saúde Colet 2012; 20(1):64-71.

48. Ajmera M, Raval A, Zhou S, Wei W, Bhattacharya R, Pan C, Sambamoorthi U. A Real-World Observational Study of Time to Treatment Intensification Among Elderly Patients with Inadequately Controlled Type 2 Diabetes Melito. J Manag Care Spec Pharm 2015; 21(12):1184-1193.

Artigo apresentado em 13/04/2016

Aprovado em 23/08/2016

Versão final apresentada em 25/08/2016 